\title{
Railway Safety Risk Assessment and Control Optimization Method Based on FTA-FPN: A Case Study of Chinese High-Speed Railway Station
}

\author{
Qi Zhang $\mathbb{D}^{\mathbb{D}},{ }^{1}$ Yanhui Zhuang, ${ }^{1,2}$ Yuguang Wei, ${ }^{1}$ Hao Jiang $\mathbb{D}^{1},{ }^{1}$ and Hao Yang $^{1}$ \\ ${ }^{1}$ School of Traffic and Transportation, Beijing Jiaotong University, Beijing 100044, China \\ ${ }^{2}$ China Civil Engineering Construction Corporation, Beijinsg 100038, China \\ Correspondence should be addressed to Hao Jiang; jiangh19950223@163.com
}

Received 28 December 2018; Revised 13 November 2019; Accepted 14 December 2019; Published 1 February 2020

Academic Editor: Eleonora Papadimitriou

Copyright (c) 2020 Qi Zhang et al. This is an open access article distributed under the Creative Commons Attribution License, which permits unrestricted use, distribution, and reproduction in any medium, provided the original work is properly cited.

In order to make safety risk assessment more accurately and more reasonably for high-speed railway station in China, this paper analyzes risk factors of fault tree and transfers the fault tree of risk accident into fuzzy petri net and then builds the FPN-FTA model by combining the dynamic weighting fuzzy petri net (FPN) and fault tree analysis (FTA) based on the latter. This paper simulates the FTA-FPN model with Stateflow of Matlab software. Then, it builds up a bi-objective risk control model, making the minimum safety risk level and minimum necessary cost as the objectives, and it designs discrete particle swarm optimization algorithm to solve the risk control model. Finally, this paper selects stampede accident of Shijiazhuang high-speed railway station as an example in case study for assessing stampede risk level and gets the risk control schemes for this station. The results verify the feasibility and validity of the model and algorithm.

\section{Introduction}

The pressure on railway transport safety management has increased constantly with the rapid development of railway. Therefore, safety risk control is the key to secure railway transport. Safety risk control refers to the scientific management methods of realizing the maximum safety guarantee with the minimum cost by controlling risks effectively and optimizing safety risk control technologies based on the assessment of safety risks during the enterprise production activities. Safety Risk Assessment and Control Optimization can not only reduce probability of risk occurrence but also keep the risk control cost within reasonable boundaries, and many scholars have done researches on this topic [1-14]. Fault tree theory is widely applied to safety risk assessment at present [5-8], while fuzzy petri net is mostly used for safety diagnosis [9-14]. However, there is no existed literature that combines the fault tree and fuzzy petri net to make railway safety risk assessmentexcept our master thesis [15].
Although these methods or models have contributed to the safety risk control theory of railway transport in different aspects, there are still several problems need to be discussed.

(1) In the previous safety risk assessment research, the indicator system is used to analyze the system as a whole, but the causal correlation of the development of security risk events is not described.

(2) The traditional studies on the safety risk of transportation system consider the ambiguity and uncertainty of risk factors not enough. It is impossible to determine the risk status of the system accurately.

(3) The weight value of each risk factor is relatively fixed, and it cannot directly reflect the influence degree of the development stage with different risk factors of the risk accident.

(4) In previous studies, the control of the risk is lack of balanced analysis between benefit and cost since there is not enough consideration for the cost control. 
In order to solve these problems, this paper takes the similar characteristics of dynamic weighting fuzzy petri net (FPN) and fault tree analysis (FTA) into consideration, introduces FTA-FPN into railway safety risk assessment, analyzes risk factors of fault tree, maps the fault tree of risk accident into fuzzy petri net, and builds up railway safety risk assessment model combined with both FPN and FTA [4]. Then, this paper proposes to simulate FTA-FPN model by Stateflow, considering the graphic characteristics of fuzzy petri net. Based on Target Cost Method, cost control is introduced into risk control optimization. A bi-objective model with the minimum safety risk level and minimum necessary cost as the objectives is built up, and corresponding discrete particle swarm algorithm is designed for solving the model. The paper makes the risk control of passenger traffic congestion in high-speed rail passenger station as an example to carry out method verification. An empirical study is conducted at the Shijiazhuang High-speed Railway Station to assess the safety risk level of the crowded stampede event and the control optimization plan.

\section{Safety Risk Assessment and Control Model of Railway Based on FTA-FPN}

2.1. General Idea. In railway system, different control methods are taken to deal with different risk factors, then different costs and contributes to system general risk level are caused. Therefore, systematic risk control aims at reducing the system risk level effectively by controlling different risk factors selectively and saving the cost. This paper builds railway safety risk assessment model combining FPN and FTA and builds the double objective optimization model of risk control with the minimum safety risk level and minimum necessary cost of safety risk control.

\subsection{Theoretical Research}

2.2.1. Fault Tree Analysis. Fault tree can be used for analyzing the relevant cause events, including their interactions and logical relations with a specific selected risk accident as the target, building up the fault tree model and analyzing the importance of cause events and finally designing the scientific safety risk control measures contrapuntally. Fault tree consists of two parts: qualitative analysis and quantitative analysis. Qualitative analysis refers to comprehensive recognition of risk factors, mapping logical graph, and finding all cause events, including their combinations. Quantitative analysis refers to analysis of the structural importance of the cause events and determination of their influences on the top event, that is, the risk accident.

2.2.2. Dynamic Weighting Fuzzy Petri Net. Dynamic weighting fuzzy petri net is a transformation of the normal petri net; it consists of places, transitions, and the flow relations between them. However, the composition and element meaning of dynamic weighting fuzzy petri net are different from the normal petri net. Dynamic weighting fuzzy petri net can be defined by a ten-element group [8]:

$$
S_{\mathrm{WFPN}}=(P, T, D, I, O, \mu, \alpha, W, \lambda, M),
$$

where

$P=\left\{p_{1}, p_{2}, \ldots, p_{n}\right\}$ refers to the nonempty set of places which represent cause events.

$T=\left\{t_{1}, t_{2}, \ldots, t_{m}\right\}$ refers to the nonempty set of transitions which represent the happening of the events.

$D=\left\{d_{1}, d_{2}, \ldots, d_{n}\right\}$ refers to the nonempty set of propositions. $|P|=|D|, P \cap T \cap D=\varnothing$.

$I: P \times T \longrightarrow\{0,1\}$ refers to the entry incidence matrix which represents the entry relations from places $p_{i}$ to transitions $t_{j} . I=\left(\theta_{i j}\right)$.

$\theta_{i j}$ refers to the input arc. If there is input arc between place $p_{i}$ and transition $t_{j}$, then $\theta_{i j}=1$, otherwise $\theta_{i j}=0$.

$O: T \times P \longrightarrow\{0,1\}$ refers to the export incidence matrix which represents the export relations from transitions $t_{j}$ to places $p_{i}$. $O=\left(\eta_{i j}\right)$.

$\eta_{i j}$ refers to the export arc. If there is output arc between transition $t_{j}$ and place $p_{i}$, then $\eta_{i j}=1$, otherwise $\eta_{i j}=0$.

$\mu: T \longrightarrow[0,1]$ refers to the real number mapping of transitions. $\mu_{j}$ is the certainty factor $(\mathrm{CF})$ of the inference rule of transition $t_{j} . \mu=\left(\mu_{1}, \mu_{2}, \ldots, \mu_{m}\right)$, $\mu_{j} \in[0,1]$.

$\alpha: P \longrightarrow[0,1]$ refers to the real number mapping of places which represent the validity of propositions $d_{i}$ that correspond to places $p_{i}$.

$P \longrightarrow D$ because the places are mapped into propositions, so places and propositions are matched one by one. $\alpha$ is the token of the petri net. $\alpha_{i}=\alpha\left(p_{i}\right)$, $\alpha_{i} \in[0,1]$.

$\lambda: T \longrightarrow[0,1]$ refers to the mapping which represents the threshold values of the triggering of transitions $t_{j}$. $\lambda_{j} \in[0,1]$.

$W=\left(\varrho_{i j}\right)$ refers to the dynamic weighting which represents the support of prerequisite proposition to result proposition; it varies with the changing of $\alpha\left(p_{i}\right)$. Dynamic weighting reflects the degree of associations between a certain indicator and other indicators in the evaluation system. The dynamic performance of the petri nets means the dynamic weight will change dynamically with the assessed value $\alpha\left(p_{i}\right)$ of each evaluation of the cause events $p_{\mathrm{i}}$ in the petri nets. Formula (2) shows the value of ${\varpi_{i j}}_{i \leq i \leq n} \Phi_{i j}=1$, $j=1,2, \ldots, m$ :

$\begin{cases}\varpi_{i j}=\frac{\theta_{i j} \alpha\left(p_{i}\right)}{\sum_{i=1}^{n} \theta_{i j} \alpha\left(p_{i}\right)}, & \theta_{i j} \neq 0, \sum_{i=1}^{n} \theta_{i j} \alpha\left(p_{i}\right) \neq 0, \\ \varpi_{i j}=0, & \theta_{i j}=0 \text { or } \sum_{i=1}^{n} \theta_{i j} \alpha\left(p_{i}\right)=0 .\end{cases}$

$M=\left(\alpha\left(p_{1}\right), \alpha\left(p_{2}\right), \ldots, \alpha\left(p_{n}\right)\right)^{T}$ refers to the identification of dynamic weighting fuzzy petri net. 
The fuzzy rules of fuzzy petri net are as follows:

(1) $\mathrm{IF} d_{i 1}$ AND $d_{i 2}$ AND $d_{i 3} \ldots$ AND $d_{i n}, \mathrm{THEN} d_{k}$ (CF $=\mu) ; \lambda ; \Phi_{1}, \Phi_{2}, \ldots, \Phi_{n}, d_{i 1}, d_{i 2}, \ldots, d_{i n}$ are prerequisite propositions and $d_{k}$ is result proposition. $\omega_{1}, \Phi_{2}, \ldots, \Phi_{n}$ represent the different supports of prerequisite propositions to result proposition. If $\varpi_{i} \in[0,1]$ and $\sum_{i=1}^{n} \alpha\left(p_{i}\right) \varpi_{i}>\lambda$, then the transition is triggered. The validity of prerequisite proposition is not changed, and the validity of result proposition $d_{k}$ is $\left|\sum_{i=1}^{n} \alpha\left(p_{i}\right) \omega_{i}\right| \mu$.

(2) $\mathrm{IF} d_{i 1} \mathrm{OR} d_{i 2} \mathrm{OR} d_{i 3} \ldots \mathrm{OR} d_{i n}, \mathrm{THEN} d_{k}\left(\mathrm{CF}_{1}=\mu_{1}\right.$ $\left., \ldots, \mathrm{CF}_{n}=\mu_{n}\right) ; \lambda_{1}, \lambda_{2}, \ldots, \lambda_{n} ; \quad \Phi_{1}, \Phi_{2}, \ldots, \Phi_{n}$. $d_{i 1}, d_{i 2}, \ldots, d_{i n}$ are prerequisite propositions; $\mu_{1}, \mu_{2}, \ldots, \mu_{n}$ are validities of different rules, $d_{k}$ is result proposition, and $\lambda_{1}, \lambda_{2}, \ldots, \lambda_{n}$ are the threshold values of the triggering of different transitions. If $\sum_{i=1}^{n} \alpha\left(p_{i}\right) \bigotimes_{i}>\lambda_{i}$, transition will be triggered. The validity of prerequisite proposition is not changed, and the validity of $d_{k}$ is $\max \left(\alpha\left(p_{i}\right) \mu_{j}\right)$.

\subsection{The Safety Risk Assessment Model of Railway System Based on FTA-FPN}

2.3.1. Railway Safety Risk Assessment Model Based on FPN and FTA. Based on the features of railway system and safety risk assessment, this paper combined the advantages of fault tree method and fuzzy petri net theory and proposed the railway safety risk assessment method based on FTA-FPN, as shown in Figure 1.

The specific rules for mapping fault tree model into fuzzy petri net model are as follows:

(1) The events of fault tree and the places of petri net can be transformed into each other. The basic events of fault tree are corresponding to initial places of petri net; the top event of fault tree is corresponding to target place of petri net.

(2) The causal relations between events of fault tree and the transitions of petri net can be transformed into each other.

(3) The logical gates mainly consist of AND gates, OR gates, NOT gates. The negativity of the event can be set as a new event, so there will be no NOT gate existing in the fault tree. The logical gates of fault tree and symbols of petri net can be transformed into each other, as shown in Figure 2.

(4) The special logical gates such as voting gates, exclusive-OR gates, and exclusion gates can be transformed into AND gates, OR gates, or their combinations. Therefore, the fault tree can only consist of AND gates and OR gates.

2.3.2. The Solving Method. There are two methods used for solving fuzzy petri net. The search algorithm based on the net model and the inference method based on the incidence matrix of petri net. The second method is popular at present for its convenience. However, it is the lack of

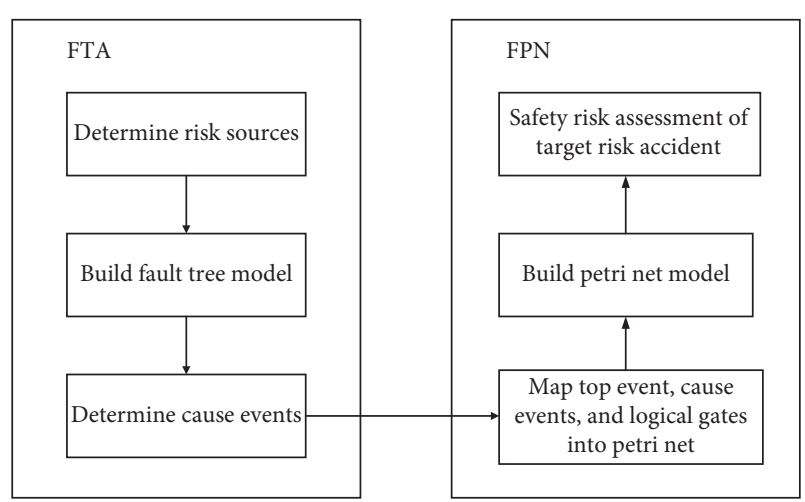

Figure 1: Process of safety risk assessment method.

efficiency while applied to large-scale net. The first method can be visualized, but it is complicated and difficult for solving manually. According to the disadvantages of the first method, this paper proposes to use Stateflow for simulating petri net model and finally solve the fuzzy petri net. Stateflow is a kind of design tool with strong visualization and integrated into Simulink of Matlab software. It is widely used for the simulation of complicated eventdriven system.

\subsection{The Safety Risk Control Model of Railway System}

\subsubsection{Assumptions}

(1) The superior limit of system risk level and the limit of total cost of risk control is both known.

(2) Cost spent on one risk source would not change the risk levels of other risk factors.

(3) The risk levels of all risk factors are known before the risk control.

(4) Leave force majeure events out of account.

\subsubsection{Parameters}

$Q$ - general risk level of the whole system

$c_{i}$-cost for controlling the risk source $i$

$Q_{m}$-maximum permissible general risk level

$C_{m}$-limit of total cost for risk control

$N$-amount of risk factors

$n$-amount of controlled risk factors

$x_{i}$-decision variable of risk source $i$

$f(x)$-risk calculation function based on the FTA-FPN model

$\alpha^{\prime}\left(p_{i}\right)$-risk level of risk source $i$ after control

$\alpha\left(p_{i}\right)$-risk level of risk source $i$ before control

2.4.3. The Railway Safety Risk Control Model. The safety risk control model with the minimum risk level $Q$ and minimum necessary cost $C$ as the objectives is built up as follows: 

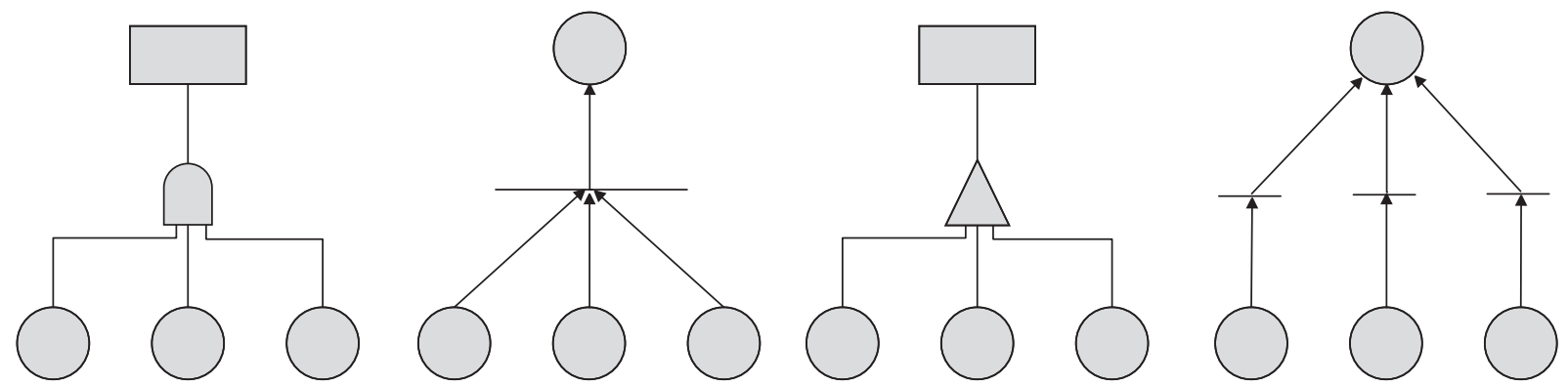

FIgURE 2: Transformation between fault tree and petri net.

$$
\begin{aligned}
& Q=\min f\left(\left(1-x_{i}\right) \alpha\left(p_{i}\right)+x_{i} \alpha^{\prime}\left(p_{i}\right)\right), \\
& C=\min \sum_{i=1}^{n} c_{i} x_{i} .
\end{aligned}
$$

Formula (3) refers to risk level of railway system, and formula (4) refers to the necessary cost for safety risk control of railway system.

Constraints:

$$
\begin{aligned}
& Q \leq Q_{m}, \\
& C \leq C_{m}, \\
& 0<n \leq N, \\
& x_{i} \in\{0,1\}, \quad i=1,2, \ldots, n, \\
& 0<\alpha^{\prime}\left(p_{i}\right) \leq \alpha\left(p_{i}\right), \quad i=1,2, \ldots, n .
\end{aligned}
$$

Formula (5) represents that the risk level of railway system cannot exceed the maximum permissible general risk level. Formula (6) represents that the cost of risk control cannot exceed the limit of total cost of risk control. Formula (7) represents that the amount of controlled risk factors cannot exceed the amount of risk factors. Formula (8) represents the decision variable of risk source $i$, if the risk source is controlled, then it is 1 , otherwise, it is 0. Formula (9) represents that risk level of risk source $i$ after control is lower than that before control.

2.5. Discrete Particle Swarm Optimization Algorithm. This paper chooses Binary Particle Swarm Optimization (BPSO), also known as Discrete Particle Swarm Optimization Algorithm, to solve the safety risk control model. BPSO belongs to Particle Swarm Optimization (PSO). It has several features as follows:

\subsubsection{Update Rule}

$$
\begin{aligned}
v_{i, l}^{\tau+1}= & \xi \times v_{i, l}^{\tau}+o_{1} \times \operatorname{Rand}() \times\left(p_{i, l}^{\tau}-x_{i, l}^{\tau}\right) \\
& +o_{2} \times \operatorname{Rand}() \times\left(p_{g, l}^{\tau}-x_{i, l}^{\tau}\right), \\
x_{i, l}^{\tau+1}= & x_{i, l}^{\tau}+v_{i, l}^{\tau+1},
\end{aligned}
$$

where $v_{i l .}^{\tau}$ is the speed of particle $i$ in dimension $l$ in iteration $\tau ; x_{i, l}^{t}$ is the position coordinates of particle $i$ in dimension $l$ in iteration $\tau$; $\xi$ is inertia weight of the current speed during state adjustment; Rand() is random coefficients evenly distributed between 0 and 1; and $o$ is acceleration limit factor. $p_{i, l}^{\tau}$ is the optimal position of particles $i$ in the population up to the $\tau$-th iteration. $p_{g, l}^{\tau}$ is the optimal position experienced by all particles in the population until the $\tau$-th iteration.

Different from basic PSO, the coding of BPSO is binary, the code of each particle is 0 or 1 . Therefore, the update rule of basic PSO is changed by introducing the fuzzy function, as formula (10) shows.

$$
\begin{aligned}
& x_{i, l}^{\tau+1}= \begin{cases}1, & \operatorname{rand}() \geq S\left(v_{i, l}^{k+1}\right), \\
0, & \operatorname{rand}()<S\left(v_{i, l}^{k+1}\right),\end{cases} \\
& S(v)=\frac{1}{1+\exp (-v)} .
\end{aligned}
$$

For basic PSO, $v_{\max }$, which is definite, is the superior limit of particle speed, while for BPSO, the particle speed $v_{i, l}^{\tau}$ can be represented in the form of probability, and $v_{\max }$ refers to the probability range of particle speed. Now, define the probability of particle position as $\operatorname{Sig}\left(v_{i, l}^{\tau}\right)=1$, then $1-\operatorname{Sig}\left(v_{i, l}^{\tau}\right)=0$. The transformation probability of particle will be

$$
\rho(\Delta)=\operatorname{Sig}\left(v_{i, l}^{\tau}\right)\left(1-\operatorname{Sig}\left(v_{i, l}^{\tau}\right)\right) .
$$

2.5.2. Inertia Weight. Inertia weight has influence on algorithm search capability. This article proposed to use nonlinear adaptive strategy for calculating the inertia weight $w$, as formula (14) shows:

$$
w=f(\tau)= \begin{cases}{\left[\frac{\left(\tau_{\max }-\tau\right)^{n}}{\left(\tau_{\max }-\tau_{\mathrm{sw}}\right)^{n}}\right]\left(w_{\mathrm{sw}}-w_{\text {final }}\right)+w_{\text {final }},} & \text { attract }=\text { true }, \\ C_{\kappa}, & \text { attract }=\text { false },\end{cases}
$$


where $\tau$ is the number of iterations at present; $\tau_{\max }$ is the maximum number of iterations; $w_{\text {initial }}$ is the initial inertia weight of particle at beginning; $w_{\text {final }}$ is the final inertia weight of particle at ending; $\tau_{\mathrm{sw}}$ is the number of iterations at present during calculation, initial value is 0 ; and $w_{\text {sw }}$ is the inertia weight at present during calculation, initial value is $w_{\text {initial }}$,

Specific calculation process:

Step 1: Initialize parameters of model, algorithm, and particle initial status such as position and speed. Calculate the sufficiency for each particle by the following formula $F(x)=\varepsilon_{1} Q(x)+\varepsilon_{2} C(x), \varepsilon_{1}=0.5$ and $\varepsilon_{2}=0.5$.

Step 2: Compare the sufficiency with the current best sufficiency Pbest of each particle; update Pbest if it is better.

Step 3: Compare the best sufficiency Pbest of each particle with the current best sufficiency Gbest of the whole group; update Gbest if it is better.

Step 4: Calculate inertia weights by formula (12), then update particle positions by formula (10), correct unsuitable particles of constraints, and generate the new particle group.

Step 5: Check the number of iterations, if it has reached the maximum number, end calculation, and turn to Step 6, otherwise, turn to Step 1.

Step 6: Output result.

\section{Case Study}

This paper uses stampede accident in high-speed railway stations as the example of method validation. Comparing with the normal railway station, high-speed railway station covers a larger area, has more equipment, and more complicated passenger flows. Stampede accident may easily occur if there is passenger tumble or escalator failure. Therefore, it is vital to control the risks of stampede accidents accurately and efficiently in the high-speed railway station.

3.1. Stampede Risk Assessment Model of High-Speed Railway Station Based on FTA-FPN. This paper builds the fault tree model of stampede accident of high-speed railway station and maps it into fuzzy petri net based on the transformation methods. Then, the stampede risk assessment model of high-speed railway station based on FTA-FPN is built. Figure 3 and Table 1 specify the contents of places. Place $P_{i}$ represents factor that may cause stampede accident, the corresponding proposition $d_{i}$ represents the status of the factor. The reliability $\alpha\left(p_{i}\right)$ of $d_{i}$ represents its assessed value under different statuses. Transition $t_{j}$ represents the development of event. Fuzzy factor $\mu_{j}$ of transition $t_{j}$ represents the possibility of development. The corresponding risk level of token value of place $P_{21}$ can represent the stampede risk level in high-speed railway station.

3.2. Stampede Risk Control Model of High-Speed Railway Station Based on FTA-FPN. The stampede risk control model of high-speed railway station based on the FPN model of stampede risk is built, and its object function and constraints are shown as follows:

$\left\{\begin{array}{l}Q_{21}=\min \left(Q_{19} \Phi_{19} \mu+Q_{20} \Phi_{20} \mu\right), \\ C=\min \sum_{i=1}^{12} c_{i} x_{i},\end{array}\right.$

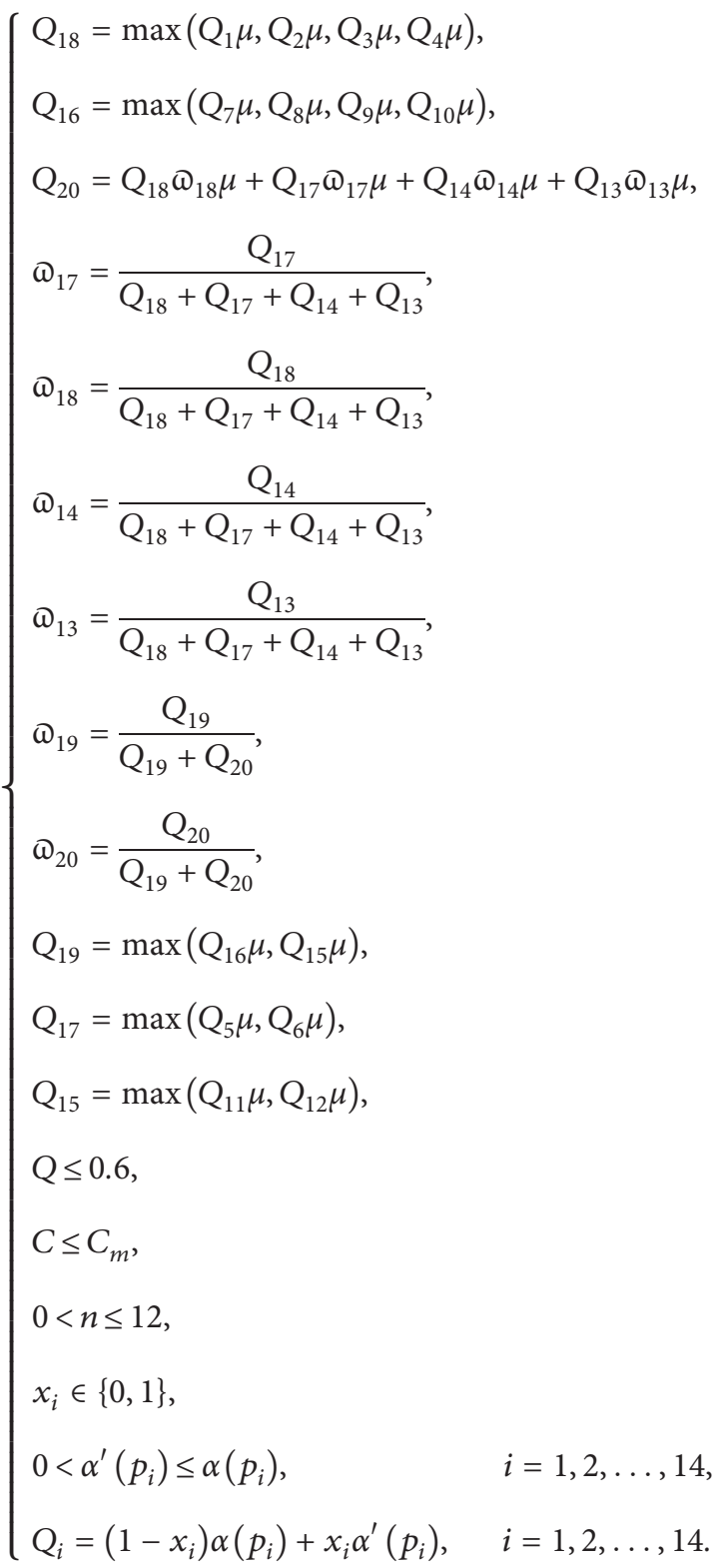




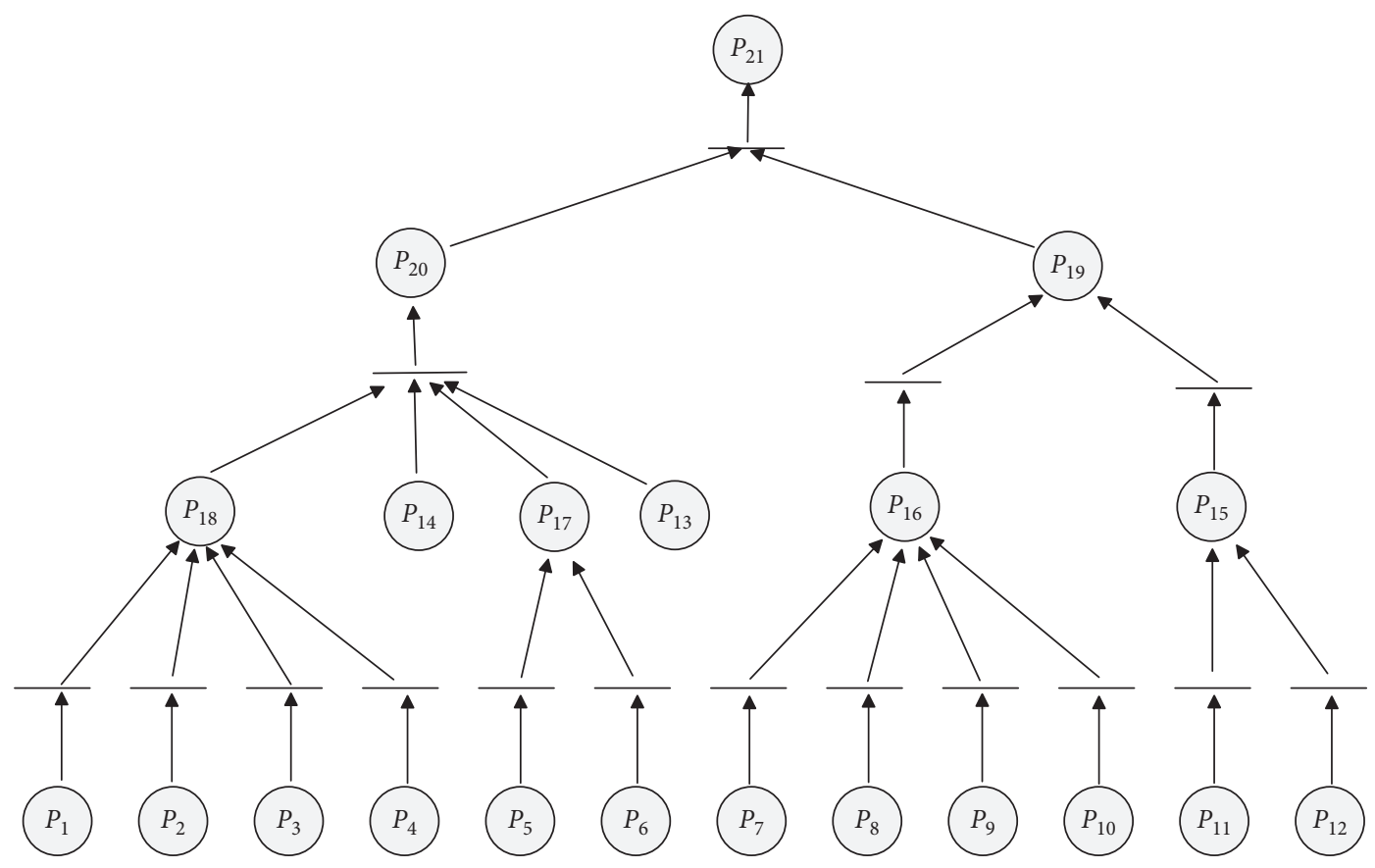

FIGURE 3: FPN model of stampede risk in high-speed railway station.

Table 1: Illustration of Places in Petri Net Model.

\begin{tabular}{lc}
\hline Place & Content \\
\hline$P_{1}$ & Crowded station entrance \\
$P_{2}$ & Crowded ticket or boarding entrance \\
$P_{3}$ & Crowded escalator or passage \\
$P_{4}$ & Crowded station exit \\
$P_{5}$ & Insufficient emergency response ability \\
$P_{6}$ & Insufficient safety awareness (staff) \\
$P_{7}$ & Fire explosion \\
$P_{8}$ & Escalator failure \\
$P_{9}$ & Terrorist attack \\
$P_{10}$ & Passenger tumble \\
$P_{11}$ & Unsuitable escalator or passage \\
$P_{12}$ & Unsuitable station entrance and exit \\
$P_{13}$ & Poor emergency evacuation \\
$P_{14}$ & Insufficient safety awareness (passenger) \\
$P_{15}$ & Poor station design \\
$P_{16}$ & Unforeseen circumstances \\
$P_{17}$ & Poor manual guidance \\
$P_{18}$ & Passenger gathering \\
$P_{19}$ & Activating event \\
$P_{20}$ & Excessive passenger density \\
$P_{21}$ & Stampede accident \\
\hline
\end{tabular}

3.3. Assessment Criteria Quantization of High-Speed Railway Station Based on Cloud Model. According to the characteristic of assessment of stampede, this paper classifies assessment criteria into 5 classifications, including Good $(H)$, Fair $(J H)$, Normal $(Z)$, Substandard $(J C)$, and Poor $(C)$. It quantifies the assessment criteria based on cloud model. 5 cloud models that are $\left\{C_{-2}, C_{-1}, C_{0}, C_{+1}, C_{+2}\right\}$ can be generated with golden section method in the universe of $U=[0,1] .5$ cloud models are corresponding to 5 classifications, and numeric characteristics of each cloud model is shown in Table 2.

$S$ experts, recorded as $E=\left\{e_{1}, e_{2}, \ldots, e_{s}\right\}$, are invited to assess stampede risk factors, and expert weights are $W^{e}=\left\{w_{1}^{e}, w_{2}^{e}, \ldots, w_{s}^{e}\right\}$. The assessment criteria of stampede accident are recorded as $Z=\left\{z_{1}, z_{2}, \ldots, z_{n}\right\}$, and the assessed values are $x_{j}(j=1,2, \ldots, n)$. All criteria are calculated based on formula (15). More than $99.73 \%$ clouds shall be situated in the range of $[E x-3 E n, E x+$ $3 E n]$ according to the $3 E n$ rule. Considering characteristics of Hyperentropy calculation, transform cloud model $C_{j}\left(E x_{j}, E n_{j}, H e_{j}\right)$ is used into interval number $[E x-3(E n+3 H e), E x+3(E n+3 H e)]$. The assessed value of interval number can be replaced by expectation of interval. In this paper, the cloud model is normal distribution. Therefore, the assessed values of criteria are $E x$.

$$
x_{j}=\sum_{i=1}^{s} w_{i}^{e} x_{i j}=Z_{j}\left(\sum_{i=1}^{s}\left(w_{i}^{e} E x_{i j}\right), \sqrt{\sum_{i=1}^{s}\left(w_{i}^{e} E n_{i j}\right)^{2}}, \sqrt{\sum_{i=1}^{s}\left(w_{i}^{e} H e_{i j}\right)^{2}}\right) .
$$


TABLE 2: Numeric characteristics of cloud models.

\begin{tabular}{lcccc}
\hline Assessment classifications & Cloud model & Expectation $E x$ & Entropy En & Hyper entropy He \\
\hline$C$ & $C_{+2}\left(E x_{+2}, E n_{+2}, H e_{+2}\right)$ & 1.000 & 0.103 & 0.013 \\
$J C$ & $C_{+1}\left(E x_{+1}, E n_{+1}, H e_{+1}\right)$ & 0.691 & 0.064 & 0.008 \\
$Z$ & $C_{0}\left(E x_{0}, E n_{0}, H e_{0}\right)$ & 0.500 & 0.039 & 0.005 \\
$J H$ & $C_{-1}\left(E x_{-1}, E n_{-1}, H e_{-1}\right)$ & 0.309 & 0.064 & 0.008 \\
$H$ & $C_{-2}\left(E x_{-2}, E n_{-2}, H e_{-2}\right)$ & 0 & 0.103 & 0.013 \\
\hline
\end{tabular}

3.4. Stampede Risk Assessment of Shijiazhuang High-Speed Railway Station. Shijiazhuang high-speed railway station is a large transportation hub with multiple transport modes, including subway, high-speed railway, normal-speed railway, and road transport.

In this paper, 15 experts are invited to participate in the evaluation work. However, considering the daily safety assessment of the high-speed railway station, the number of experts participating in the evaluation is limited. In order to make the evaluation results be in line with the actual work, the authors selected 3 typical experts and their evaluation results for Shijiazhuang highspeed railway station. One of them is an expert on the field of passenger safety supervision for high-speed railway passenger stations with rich supervision experience. One is a senior management with high management experience in high-speed railway passenger transportation. The other is a professor of high-speed railway passenger safety management with rich theoretical knowledge. For the opinions of the other 12 experts, we have summarized their opinions with the 3 typical expert opinions, which are selected to a certain degree, and finally form an evaluation index system for the thesis. Taking into account the equality of experts from different fields, the paper sets their weights as $W^{\varepsilon}=\{1 / 3,1 / 3,1 / 3\}$.

After calculation, the assessed values of criteria can be received, as shown in Table 3.

Based on the relations between criteria and fuzzy petri net, Stateflow is used to simulate the fuzzy petri net model of stampede risk in Shijiazhuang high-speed railway station; The Stateflow model is shown in Figure 4. After substituting the assessed values of criteria, the risk levels of some risk factors can be received, as shown in Figure 5.

The risk status can be divided into 4 segments according to relevant Chinese national standards, including safe, alert, dangerous, and extreme dangerous, and the corresponding ranges of assessed value are shown in Table 4 based on the literature $[4,9]$. Calculation results show that risk status of stampede accident is alert. Main causes include crowded station entrance, escalator, or passage and two escalator failures during the assessment.

After simulation, the result is shown in Figure 4. The red square frames represent that the risk levels of the
TABle 3: Assessed values of criteria.

\begin{tabular}{lc}
\hline Criteria & $\alpha_{k}\left(p_{i}\right)$ \\
\hline$P_{1}$ & 0.691 \\
$P_{2}$ & 0.564 \\
$P_{3}$ & 0.564 \\
$P_{4}$ & 0.691 \\
$P_{5}$ & 0.436 \\
$P_{6}$ & 0.436 \\
$P_{7}$ & 0.206 \\
$P_{8}$ & 0.627 \\
$P_{9}$ & 0.103 \\
$P_{10}$ & 0.5 \\
$P_{11}$ & 0.309 \\
$P_{12}$ & 0.309 \\
$P_{13}$ & 0.309 \\
$P_{14}$ & 0.5 \\
\hline
\end{tabular}

corresponding risk factors have reached the alert level, which is 0.6 , and the control methods are necessary.

The risk levels of risk factors of stampede accident in the Shijiazhuang high-speed railway station can be received, as shown in Figure 6. The risk statuses of risk factors $P_{18}, P_{19}$, $P_{16}, P_{1}, P_{4}$, and $P_{8}$ are alert. The risk statuses of risk factors $P_{2}$ and $P_{3}$ are nearly dangerous.

The control methods for risk factors are shown in Table 5. Fire explosion $P_{7}$ and terrorist attack $P_{9}$ are left out of consideration for their assessed values are very low.

Different control methods need different amount of staff or materials, which leads to different costs. Therefore, this paper determines the costs of different control methods for different risk factors based on investigation and survey, which is shown in Table 6.

$\alpha^{\prime}\left(p_{i}\right)$ are the predicted risk levels after control, assessed by experts. The model is solved by discrete particle swarm optimization algorithm; initialization is necessary at the beginning. Population size $N=20$, study factor $o_{1}=o_{2}=$ $1.52, W_{\text {inital }}=0.9, W_{\text {final }}=0.4, C_{\kappa}=0.8$. Matlab is used for programming.

This paper sets 3 different cost constraints, including 700,000 Yuan/year, 900,000 Yuan/year, and 1150,000 Yuan/year for comparing different control results. The control results under different cost constraints are shown in Table 7. 


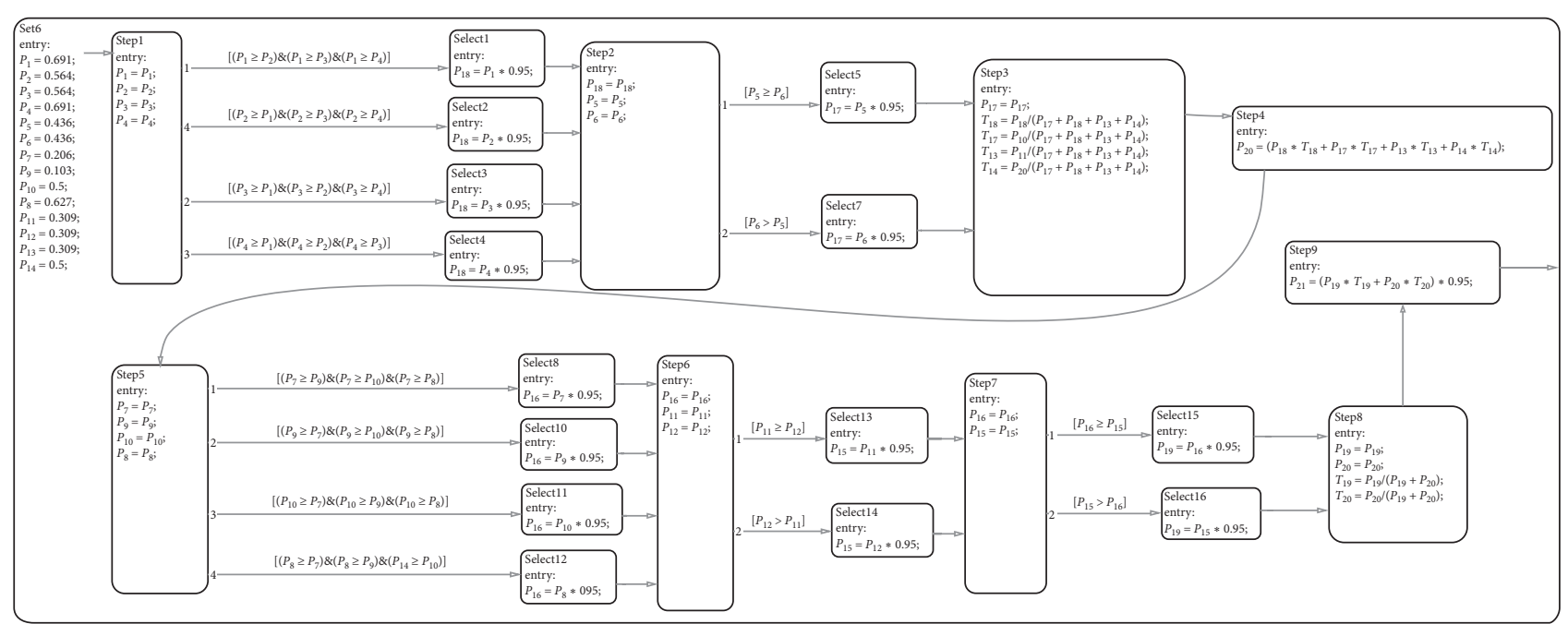

Figure 4: Stateflow model of stampede accident in Shijiazhuang station.

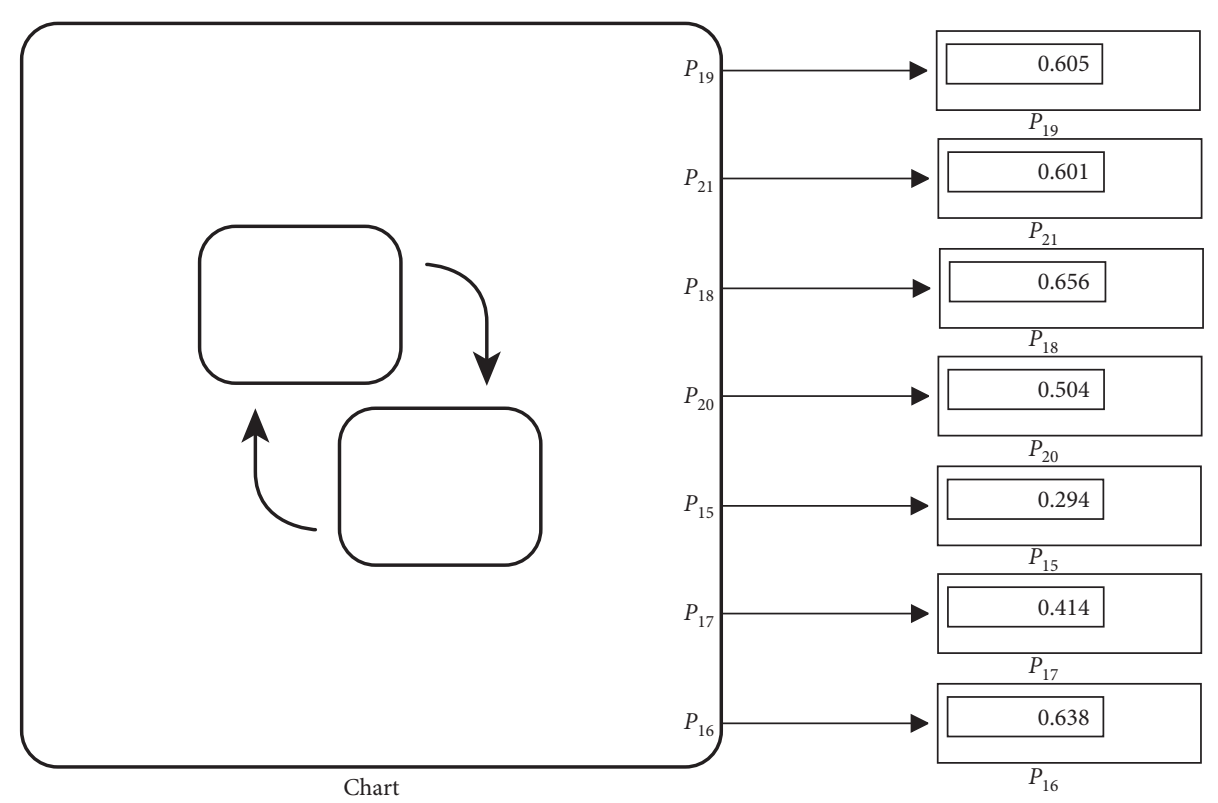

FIGURE 5: Stateflow calculation result.

TABle 4: Partitioning segments of risk status.

\begin{tabular}{lc}
\hline Risk status & Range of value \\
\hline Safe & $0,0.6$ \\
Alert & $0.6,0.7$ \\
Dangerous & $0.7,0.9$ \\
Extreme dangerous & $0.9,1.0$ \\
\hline
\end{tabular}

Figure 7 shows the control results of different risk factors under different cost constraints. Although the cost is 900,000 Yuan/year, the result is obvious, which is 0.4143 , and all risk factors remain in safe status. If safety redundancy is needed, that is, risk levels of all risk factors should remain under 0.5, and then, the control cost should be 1150,000 Yuan/year. This paper concludes that Scheme 2 is preferable under overall consideration.

\section{Conclusions}

This paper analyzes the relevant cause events of risk accidents, including their interactions and logical relations with fault tree model and then maps fault tree model into fuzzy petri net model. Risk control model with the minimum 


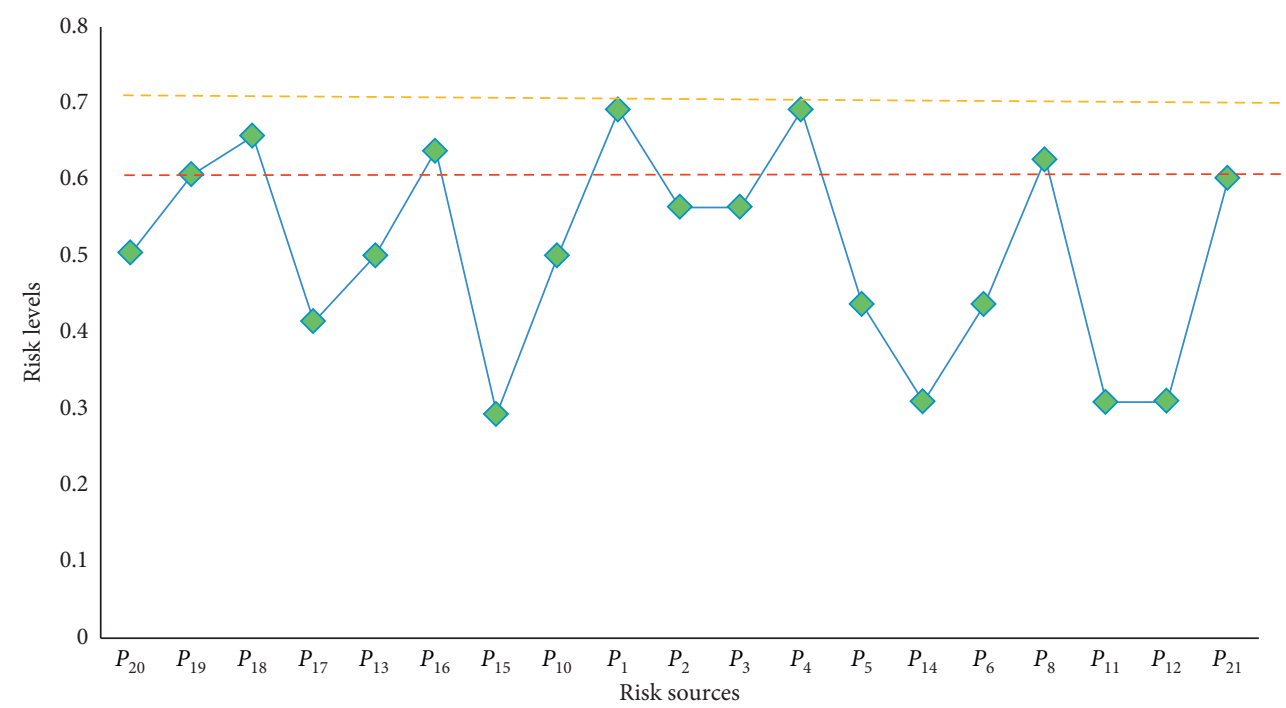

FIgURE 6: Risk levels of risk factors of stampede accident in the Shijiazhuang station.

TABLE 5: Control Methods for Risk factors.

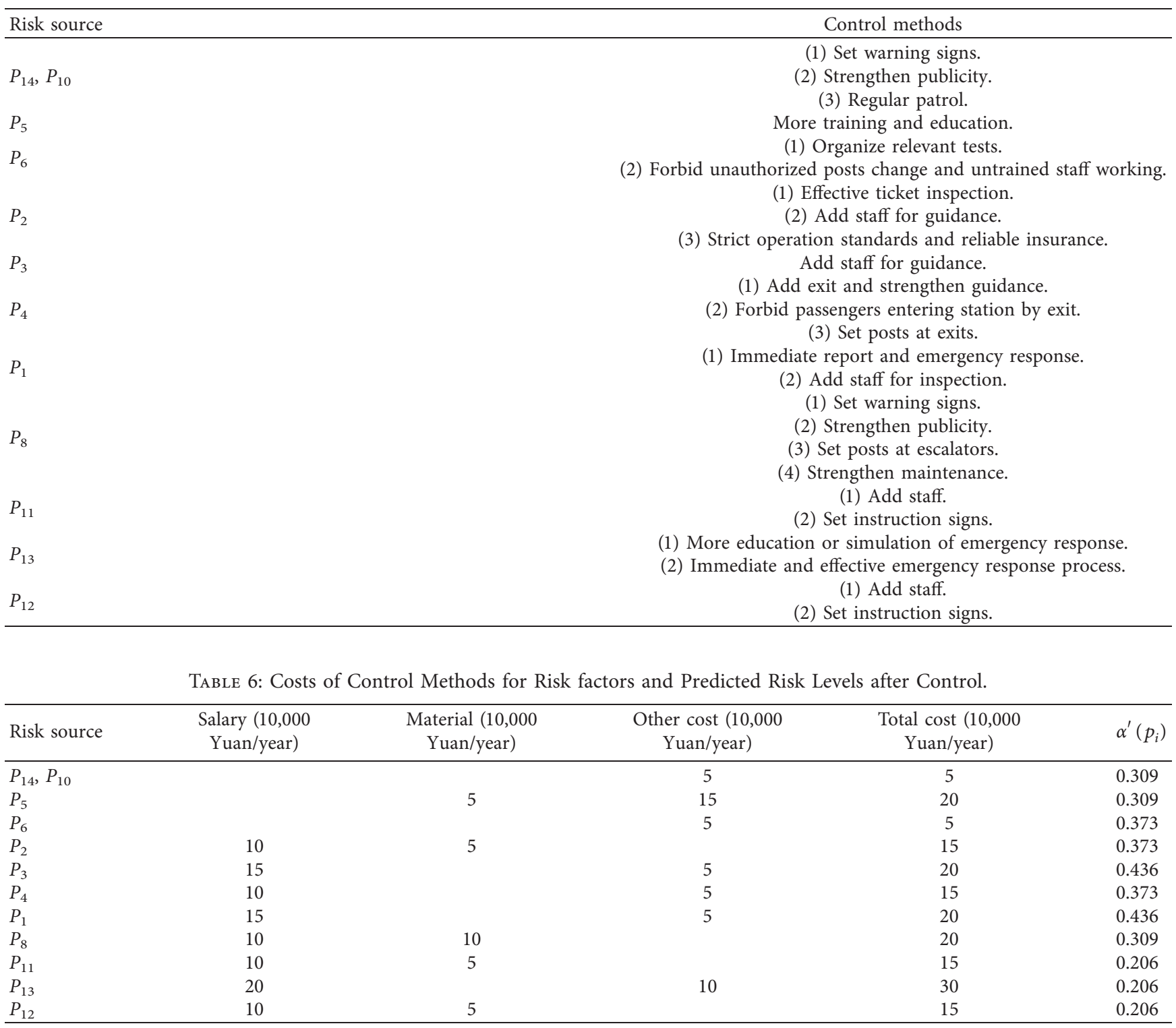


TABLE 7: Control results under different cost constraints.

\begin{tabular}{lccc}
\hline Scheme & Controlled risk source & Cost constraint & Risk level of $P_{1}$ \\
\hline 1 & $P_{1}, P_{2}, P_{3}, P_{4}$ & 700,000 Yuan/year & 0.5090 \\
2 & $P_{1}, P_{2}, P_{3}, P_{4}, P_{8}$ & 900,000 Yuan/year & 0.4143 \\
3 & $P_{13}, P_{10}, P_{1}, P_{2}, P_{3}, P_{4}, P_{5}, P_{8}$ & 1150,000 Yuan/year & 0.3801 \\
\hline
\end{tabular}

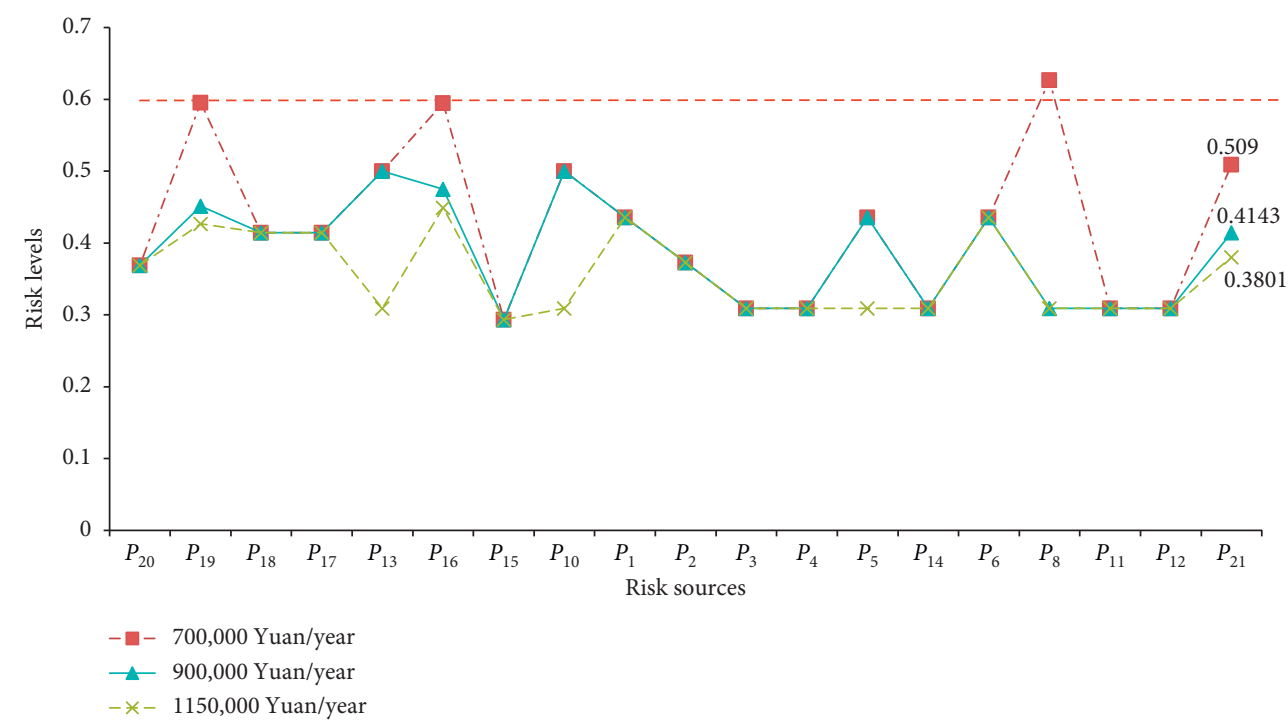

FIgURE 7: Control results of risk factors under different cost constraints.

safety risk level and minimum necessary cost as the objectives is also built up with the risk control costs as constraints. Discrete particle swarms optimization algorithm is designed accordingly. Meanwhile, Stateflow of Matlab software is used for simulating fuzzy petri net and visualized simulation for risk assessment model based on FTA-FPN is also achieved.

This paper selects stampede accident of the Shijiazhuang high-speed railway station as an example in case study for assessing stampede risk level and receiving risk control schemes. Results show that the risk level of Shijiazhuang high-speed railway station is 0.601 which is alert. Then, research for safety risk control was carried out, and results show that while the limit of total risk control cost is 900,000 Yuan/year, risk source $P_{1}, P_{2}, P_{3}, P_{4}$, and $P_{8}$ should be controlled, and the risk level shall be reduced to 0.4143 . The improvement is obvious and risk levels of all risk factors remain safe.

\section{Data Availability}

Major part of the data used to support the findings of this study are included within the article. Further information can be obtained from the author Mr. Zhuang.

\section{Conflicts of Interest}

The authors declare that they have no conflicts of interest.

\section{Acknowledgments}

This research is supported by the Chinese National Railway Administration Project, titled "Research on Safety Monitoring of High-speed Railway Passenger Transport" (T17DJ00071), and 2016 China National Key Research and Strategic International Science and Technology Innovation Cooperation Project, titled "'the Belt and Road' Land Transport International Intermodal Research and Exchange Center” (2016YFE0201700).

\section{References}

[1] H. Luo, Z. Yu, and J. Ning, "Research on railway risk control method and its application," Journal of Computer Applications, vol. 26, no. 4, pp. 40-47, 2017.

[2] J. Zhang and L. Liu, "Research on high-speed railway station safety management based on risk control theory," Journal of Transportation Engineering and Information, vol. 11, no. 4, pp. 99-104, 2013.

[3] Z. An, X. Dai, and J. Liu, "Study on safety risk control of highspeed railway," Railway Transport and Economy, vol. 36, no. 1, pp. 22-27, 2014.

[4] Y. Zhuang, Study on Safety Risk Assessment and Control Optimization of Passenger Transport in High-Speed Railway Passenger Station Based on Fuzzy Petri Net Theory, Beijing Jiaotong University, Beijing, China, 2018.

[5] J. Liu, "Synthesized risk assessment method for railway safety based on FTA-AHP analysis," China Railway Science, vol. 38, no. 2, pp. 138-144, 2017. 
[6] G. Zhan, X. Shang, and L. Hai, "AHP-based safety assessment model for rail transit system," China Railway Science, vol. 32, no. 3, pp. 123-125, 2011.

[7] J. Liu, X. Dai, G. Zhan et al., "Quantitative safety assessment method based on risk in railway system," China Railway Science, vol. 30, no. 5, pp. 123-127, 2009.

[8] E. Jafarian and M. A. Rezvani, "Application of fuzzy fault tree analysis for evaluation of railway safety risks: an evaluation of root causes for passenger train derailment," Proceedings of the Institution of Mechanical Engineers, Part F: Journal of Rail and Rapid Transit, vol. 226, no. 1, pp. 14-25, 2012.

[9] P. Ghu, L. Liu, and J. Yin, "On the subway fire risk assessment based on the dynamically variable weight petri net," Journal of Safety and Environment, vol. 16, no. 6, pp. 39-44, 2016.

[10] P. Ghu and L. Liu, "Safety assessment for integrated transportation hub based on variable weight fuzzy petri net," Journal of XIhua University (Natural Science), vol. 35, no. 5, pp. 13-17, 2016.

[11] A. Giua and C. Seatzu, "Modeling and supervisory control of railway networks using petri nets," IEEE Transactions on Automation Science and Engineering, vol. 5, no. 3, pp. 431-445, 2008.

[12] J. He, D. Zhuang, X. Zhang et al., "Study on safety of fire accident in urban mass transit system based on FTA-petri net," China Safety Science Journal, vol. 19, no. 10, pp. 77-177, 2009.

[13] D. Zhang and J. He, "Constructing security model of vehicle in urban mass transit system based on petri net," Journal of Transportation Engineering and Information, vol. 7, no. 4, pp. 109-113, 2009.

[14] P. Lei, The Method Study of Fuzzy Petri Nets Based Accident Causation analysis, Guangdong University of Technology, Guangdong, China, 2015.

[15] J. Zhang, Research on Risk Management and Evaluation Model of Grid Enterprise Informatization Project, North China Electric Power University, Beijing, China, 2015. 